Article

\title{
A Real-Time Optimal Car-Following Power Management Strategy for Hybrid Electric Vehicles with ACC Systems
}

\author{
Xiaobo Sun ${ }^{1,2}$, Weirong Liu ${ }^{1, *}$, Mengfei Wen ${ }^{3}$, Yue $\mathrm{Wu}^{2}$, Heng $\mathrm{Li}^{1}{ }^{1}$, Jiahao Huang ${ }^{2}$, Chao $\mathrm{Hu}^{4}$ and Zhiwu Huang ${ }^{2}$ \\ 1 School of Computer Science and Engineering, Central South University, Changsha 410083, China; \\ 184611050@csu.edu.cn (X.S.); liheng@csu.edu.cn (H.L.) \\ 2 School of Automation, Central South University, Changsha 410083, China; 184601020@csu.edu.cn (Y.W.); \\ 194611031@csu.edu.cn (J.H.); hzw@csu.edu.cn (Z.H.) \\ 3 Changsha College for Preschool Education, Changsha 410007, China; wmfdcf@126.com \\ 4 Big Date Institute, Central South University, Changsha 410087, China; huchao@csu.edu.cn \\ * Correspondence: frat@csu.edu.cn
}

Citation: Sun, X.; Liu, W.; Wen, M.; Wu, Y.; Li, H.; Huang, J.; Hu, C.; Huang, Z. A Real-Time Optimal Car-Following Power Management Strategy for Hybrid Electric Vehicles with ACC Systems. Energies 2021, 14, 3438. https://doi.org/10.3390/ en14123438

Academic Editor: Aldo Sorniotti

Received: 24 March 2021

Accepted: 2 June 2021

Published: 10 June 2021

Publisher's Note: MDPI stays neutral with regard to jurisdictional claims in published maps and institutional affiliations.

Copyright: (c) 2021 by the authors. Licensee MDPI, Basel, Switzerland. This article is an open access article distributed under the terms and conditions of the Creative Commons Attribution (CC BY) license (https:// creativecommons.org/licenses/by/ $4.0 /)$.

\begin{abstract}
This paper develops a model predictive multi-objective control framework based on an adaptive cruise control (ACC) system to solve the energy allocation and battery state of charge (SOC) maintenance problems of hybrid electric vehicles in the car-following scenario. The proposed control framework is composed of a car-following layer and an energy allocation layer. In the car-following layer, a multi-objective problem is solved to maintain safety and comfort, and the generated speed sequence in the prediction time domain is put forward to the energy allocation layer. In the energy allocation layer, an adaptive equivalent-factor-based consumption minimization strategy with the predicted velocity sequences is adopted to improve the engine efficiency and fuel economy. The equivalent factor reflects the extent of SOC variation, which is used to maintain the battery SOC level when optimizing the energy. The proposed controller is evaluated in the New York City Cycle (NYCC) driving cycle and the Urban Dynamometer Driving Schedule (UDDS) driving cycle, and the comparison results demonstrate the effectiveness of the proposed controller.
\end{abstract}

Keywords: connected hybrid electric vehicle; energy management; receding horizon control; energysaving

\section{Introduction}

With the growing energy shortages and environmental pollution, the development of new energy vehicles is getting more and more attention in the world. New energy vehicles mainly include electric vehicles (EVs), fuel cell vehicles, hybrid electric vehicles (HEVs), etc. [1]. Hybrid electric vehicles can provide extra electric energy and improve the engine's fuel efficiency compared to conventional cars, and effectively avoid the problem of the short cruising range of electric vehicles. HEVs combine the advantages of electricity and fuel to achieve both energy savings and emissions reduction [2].

Car-following mode is quite common in the real traffic scenarios [3]. However, it is difficult for a human driver to maintain the following performance and optimize the energy efficiency concurrently. Recently, although not universally, vehicle manufacturers have started to offer ACC systems to improve the road traffic conditions, reduce driver fatigue, improve passenger comfort [4], and so on. Common ACC methods include proportional integral derivative (PID) control, sliding mode control (SMC), and model predictive control (MPC) methods [5].

Among the common ACC methods, the PID control method works well in local control, while it is easily affected by changes in the system parameters and load [6]. The sliding mode control method is more robust than the PID control method, but chattering is inevitable. The model predictive control method can obtain the optimal control under certain constraints, and therefore can keep up with the leading car quickly while maintaining a safe distance in the car-following control [7]. 
Additionally, model predictive control can successfully realize energy optimization while keeping a safe distance in car-following scenarios. An integrated model predictive control method is proposed in [8] that combines power management and adaptive velocity control during vehicle-following scenarios. Hu et al. developed an MPC-based multi-objective framework for HEVs in car-following scenarios to investigate the interplay between fuel economy, vehicle exhaust emissions and driving safety [9]. Xie et al. proposed a pragmatic low-cost model predictive control management strategy for a plug-in hybrid electric bus by simultaneously considering energy consumption cost and computational efficiency [10]. Li et al. proposed a modified MPC method to integrate the driver's behavior into the energy management strategy [11]. Therefore, model predictive control is suitable for the energy efficiency optimization of hybrid electric vehicles in car-following scenarios.

Much research has been done in the study of HEV energy management. As it coordinates the output power of the motor and the engine, the energy management strategy is of great significance for improving the economy and efficiency of hybrid electric vehicles, and is a focus of HEV research [12]. Common energy management strategies for hybrid electric vehicles can be divided into rule-based strategies and optimization-based strategies [13].

Rule-based control strategies have been widely used in hybrid vehicle controllers. According to the different setting rules, these strategies can be divided into logic threshold control strategies and fuzzy logic control strategies. The logic threshold control strategy mainly determines the corresponding output variables by setting different thresholds and rules, so that the operating point of the engine is in a high-efficiency area [14]. Then, the genetic algorithm can be used to optimize the membership function, whose effectiveness is proved by comparing the rule-based control strategies [15]. However, these rule-based strategies rely heavily on human experience, and it is difficult to adapt to different road conditions [16].

The optimization-based methods are often divided into global optimization and instantaneous optimization. Global optimization algorithms are implemented based on the entire driving cycle [17]. Global optimization algorithms generally include a dynamic programming algorithm (DP) and Pontryagin's minimum principle (PMP). The global optimization algorithm generally needs to know the global operating conditions in advance [18], which limits the practical applications of the global optimal algorithms.

Currently, the research on hybrid electric vehicle energy management strategies focuses on the instantaneous optimization of energy management control strategies, mainly including model predictive control and equivalent fuel consumption minimization strategy (ECMS) [19]. By introducing an equivalent factor, Barsali et al. equate the consumed electric energy to fuel consumption plus engine fuel equivalent as the objective function for optimization, and use ECMS to optimize the objective function [20]. Zhang et al. adopt a fuzzy PI controller for hybrid electric vehicles to adjust the equivalent factor in real time, which realizes the control effect with strong robustness [21]. Xie et al. design an artificial neural network to dynamically identify equivalent factors based on the current demand power, the ratio of driving distance to total distance, and SOC status [22]. However, the above work mainly focuses on energy optimization. For hybrid electric vehicles, the protection of batteries is also important, and the energy management strategies should avoid the rapid degeneration of batteries.

In this paper, we propose a control strategy for optimizing the battery energy of a hybrid electric vehicle traveling in the car-following condition. Different from the existing work $[23,24]$, the SOC of the batteries of the hybrid electric vehicle is almost stable during the entire trip when optimizing the batteries' energy. In our work, the hierarchical control strategy is divided into two control layers. Both layers are based on an MPC framework but the solving methods are different. On the high layer, the car-following layer adopts sequential quadratic programming (SQP) algorithm to solve the MPC and to generate the predicted speed profiles of hybrid electric vehicles. The SQP algorithm is an effective algorithm for nonlinear constrained optimization problems, by which the original optimization is divided into a series of quadratic programming sub-problems. On the lower layer, the 
energy allocation layer, ECMS strategy is adopted to allocate energy between engine and battery in real-time.

There are three contributions in this paper.

(1) This paper proposes a hierarchical framework that integrates car-following control optimization goals and hybrid energy storage optimization goals to achieve the goal of joint optimization.

(2) The SQP algorithm is adopted to solve the optimization problem in the car-following layer, with the advantage of reducing computational overhead and achieving real-time performance.

(3) The ECMS energy management strategy can not only make full use of the energy of the energy storage device, but also maintain the battery SOC level. It is also helpful for reducing fuel consumption and emissions.

The rest of this paper is organized as follows. In Section 2, the model of the hybrid electric vehicle is constructed. In Section 3, the hierarchical control strategy is formulated. Section 4 presents comparative simulation results to verify the proposed algorithm. Section 5 provides conclusions.

\section{Model of the Hybrid Electric Vehicles}

In this section, the car-following scenario and the drive system of the hybrid electric vehicle are constructed. In the car-following scenario, the following vehicle is following the leading vehicle while keeping a safe distance (Figure 1). The safe following distance can be preset manually according to the driver's experience and the vehicle type. The drive system model consisting of the engine, battery, clutch, motor and automatic mechanical transmission (AMT) is shown in the Figure 2.

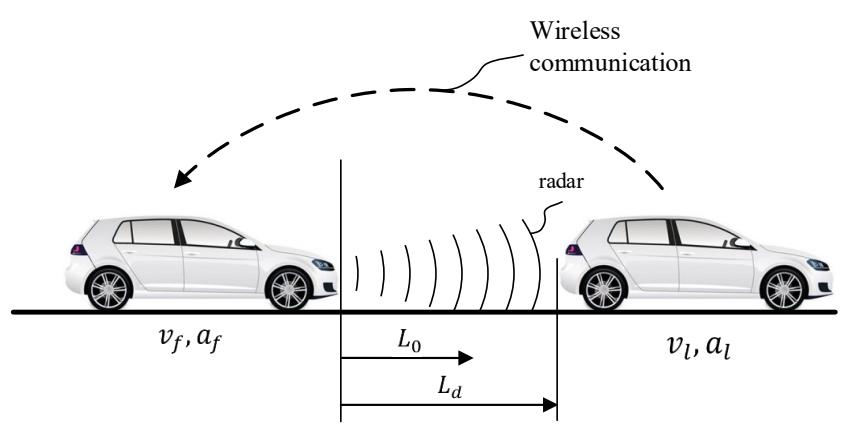

Figure 1. Scheme of the car-following scenario in this paper.

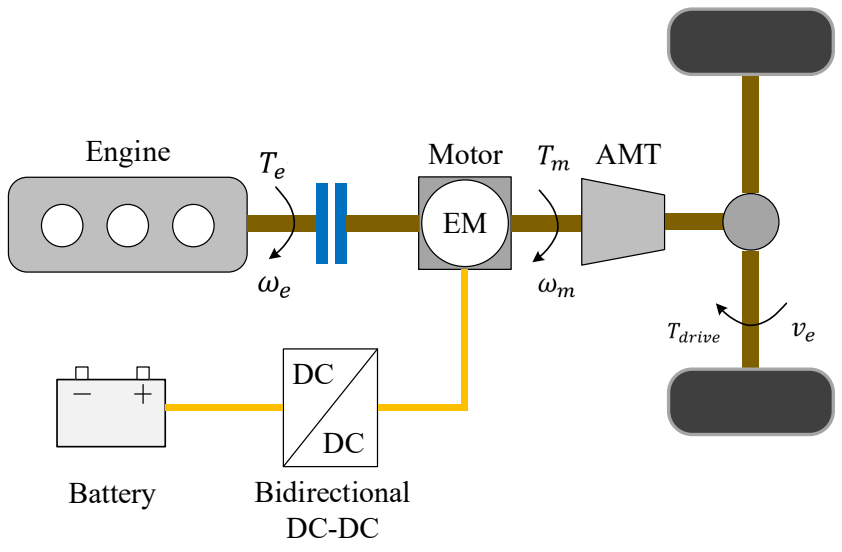

Figure 2. The structure of the hybrid electric vehicle. 


\subsection{Longitudinal Dynamics of the Following Vehicle}

The displacement and speed of the following vehicle can be expressed as follows according to [9]:

$$
\begin{gathered}
d_{f, k+1}=d_{f, k}+v_{f, k} \cdot T_{s}+\frac{1}{2} \cdot a_{f, k} \cdot T_{s}^{2} \\
v_{f, k+1}=v_{f, k}+a_{f, k} \cdot T_{s}
\end{gathered}
$$

Here, the symbol $f$ represents the following vehicle, $k$ is the time step, $d_{f, k}$ is the displacement of the following vehicle at $k, d_{f, k+1}$ is the displacement of the following vehicle at $k+1, v_{f, k}$ is the velocity of the following vehicle at $k, v_{f, k+1}$ is the velocity of the following vehicle at $k+1, T_{s}$ is the sampling time, and $a_{f, k}$ is the acceleration of the following vehicle at $k$.

The following vehicle's torque on the wheels can be obtained as

$$
T_{d}=\left(m g C_{r} \cos \theta+m g \sin \theta+\frac{1}{2} \rho A C_{D} v_{f}^{2}+m a_{f}\right) \cdot r_{w}
$$

Here, $C_{r}$ is the rolling resistance coefficient, $C_{D}$ is the aerodynamic drag coefficient. $\rho$ is the air density, $A$ is the frontal area, $\theta$ is the road slope and $r_{w}$ is the wheel radius.

For the hybrid electric vehicle in this paper, wheel torque consists of the engine torque, the motor torque and the braking torque [9].

$$
\begin{gathered}
T_{d}=\left(T_{\text {eng }}+T_{\text {mot }}\right) i_{g} i_{0} \eta_{T}^{\operatorname{sign}\left(T_{\text {mot }}\right)}+T_{\text {brk }} \\
\operatorname{sign}\left(T_{\text {mot }}\right)=\left\{\begin{array}{cc}
1 & T_{\text {mot }}>0 \\
-1 & T_{\text {mot }}<0
\end{array}\right.
\end{gathered}
$$

Here, $T_{e n g}$ is the engine torque and $T_{e m}$ is the motor torque. $i_{g}$ is the ratio of AMT, $i_{0}$ is the ratio of the differential. $\eta_{T}$ denotes the transmission efficiency.

\subsection{Engine Fuel Consumption Model}

In this paper, experimental modeling is adopted without considering the dynamic process of the engine. The fuel consumption rate corresponding to different speeds and torques is obtained by fitting the test data based on the fuel consumption data measured by a steadystate engine experiment. A brake-specific fuel consumption (BSFC) map $(\mathrm{g} / \mathrm{kWh})$ is shown in Figure 3 (https://ww2.mathworks.cn/help/autoblks/ref/mappedsiengine.html?s_tid= srchtitle (accessed on 23 March 2021)). Therefore, the fuel consumption $m_{f c}(\mathrm{~g} / \mathrm{s})$ can be calculated with the current engine torque and speed by consulting the map.

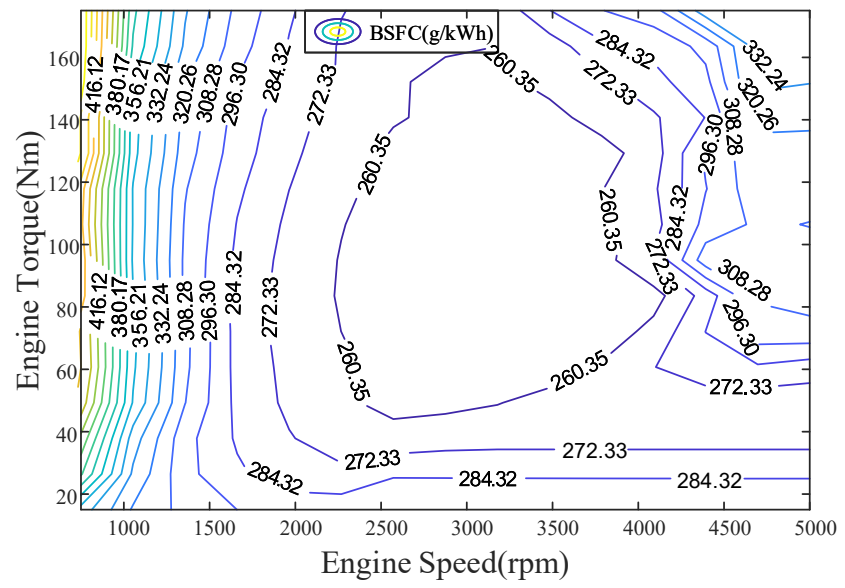

Figure 3. Brake-specific fuel consumption (BSFC) map of the engine. 


$$
m_{f c}=\frac{P_{\text {eng }} \cdot Q_{B S F C}\left(T_{\text {eng }}, \omega_{\text {eng }}\right)}{3.6 \times 10^{3}}
$$

Here, $P_{\text {eng }}$ represents the engine power $(\mathrm{kW}), T_{\text {eng }}$ is the engine torque $(\mathrm{Nm}), \omega_{\text {eng }}$ is the engine speed $(\mathrm{r} / \mathrm{min})$ and $Q_{B S F C}$ is the fuel consumption value obtained by consulting the map.

\subsection{Electric Motor Model}

When the vehicle is running normally, the driving power demand is provided by the engine and the battery at the same time. When the vehicle is braking, the motor can be used as a generator to convert the regenerative braking energy generated during braking into electrical energy to charge the battery $[25,26]$. An efficiency map of the electric motor is shown in Figure 4.

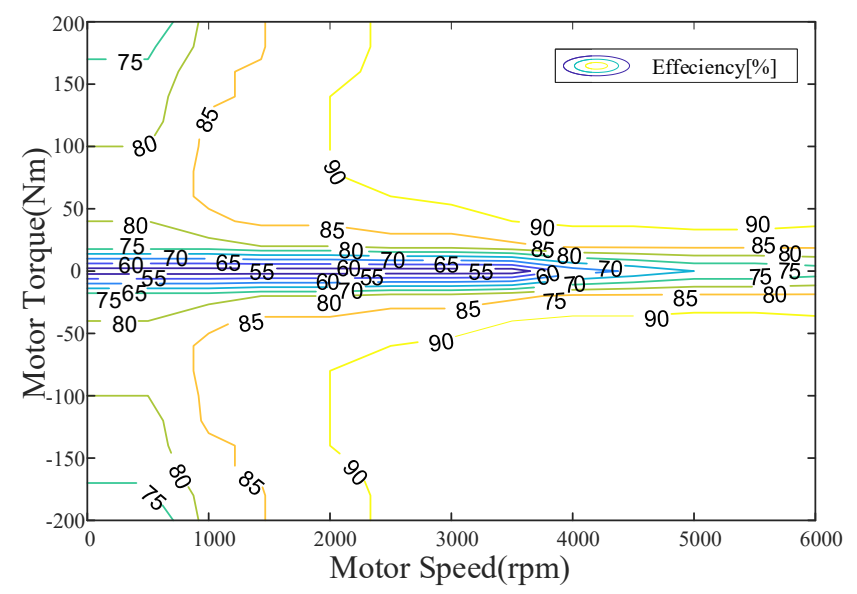

Figure 4. Efficiency map of the electric motor.

The motor power $P_{m o t}$ can be obtained by the following formula:

$$
P_{m o t}=T_{m o t} \cdot \omega_{m o t}
$$

In this formula, $P_{\text {mot }}$ is motor power (W), $T_{\text {mot }}$ is motor torque $(\mathrm{Nm})$ and $\omega_{\text {mot }}$ is motor speed ( $\mathrm{rad} / \mathrm{s})$. In our paper, the unit of motor power $P_{\text {mot }}$ is $(\mathrm{kW})$ and the unit of motor speed $\omega_{\text {mot }}$ is $(\mathrm{r} / \mathrm{min})$, so motor power $P_{\text {mot }}$ can be converted as:

$$
P_{m o t}=\frac{2 \pi}{6000} T_{m o t} \cdot \omega_{m o t}
$$

Then, we can get the relationship between battery power and motor torque, speed and efficiency as in Equation (9):

$$
P_{\text {bat }}\left(\omega_{\text {mot }}, T_{\text {mot }}\right)= \begin{cases}\frac{T_{\text {mot }} \omega_{\text {mot }}}{9550 \eta_{\text {mot }}} & T_{\text {mot }}>0 \\ \frac{T_{\text {mot }} \omega_{\text {mot }} \eta_{\text {mot }}}{9550} & T_{\text {mot }} \leq 0\end{cases}
$$

Here, $P_{b a t}$ is the battery power and $\eta_{m}$ is the motor efficiency.

The equivalent fuel consumption of the battery according to reference [27] can be expressed as

$$
m_{b a t}=\frac{\bar{Q}_{B S F C} \cdot P_{b a t}}{3.6 \times 10^{3}}
$$

Here, $\bar{Q}_{B S F C}$ is the average fuel consumption rate, and its value is set to $240 \mathrm{~g} /(\mathrm{kWh})$. $m_{b a t}$ represents the equivalent fuel consumption of the battery $(\mathrm{g} / \mathrm{s})$. 


\subsection{Battery Model}

In this paper, the internal resistance model is used to construct the battery equivalent circuit model, without considering the influence of temperature on battery performance [26]. The dynamic equation of the battery is as follows:

$$
S o C_{k+1}=S o C_{k}-\frac{V_{b a t}-\sqrt{V_{b a t}^{2}-4 R_{\text {in }} P_{b a t, k}}}{2 R_{\text {in }} Q_{\text {bat }}} \cdot T_{S}
$$

Here, $V_{b a t}$ is the open-circuit voltage, $R_{i n}$ is the battery internal resistance and $Q_{b a t}$ is the battery capacity.

\section{Control Strategy}

An overview of the hierarchical control scheme can be seen in Figure 5. It consists of a car-following layer and an energy allocation layer. The load power of the following vehicle can be calculated from the car-following layer, and the energy allocation layer can optimize the power allocation in real-time.

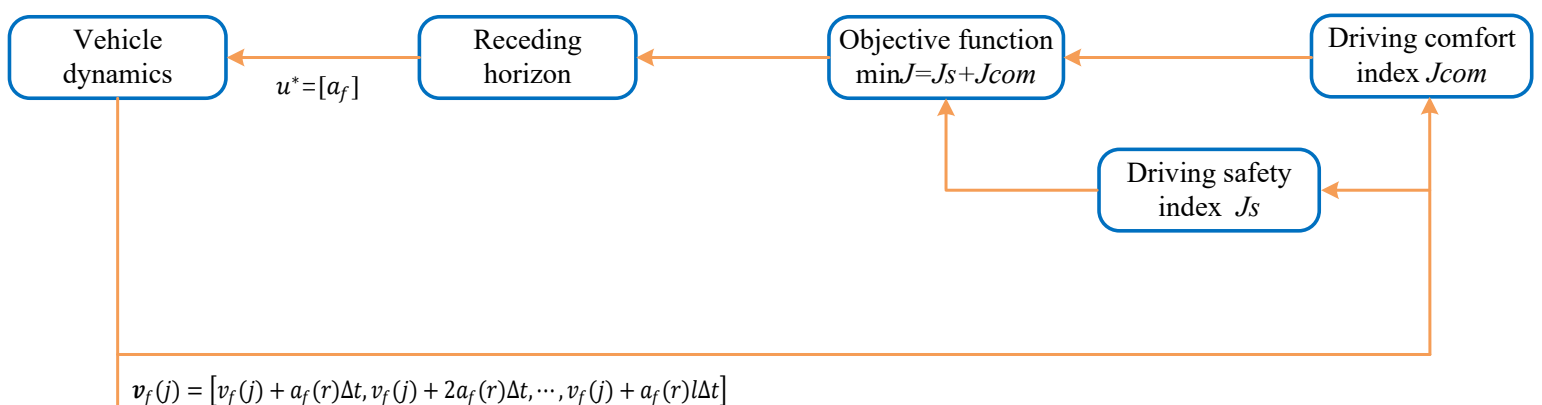

$v_{f}(j)=\left[v_{f}(j)+a_{f}(r) \Delta t, v_{f}(j)+2 a_{f}(r) \Delta t, \cdots, v_{f}(j)+a_{f}(r) l \Delta t\right]$

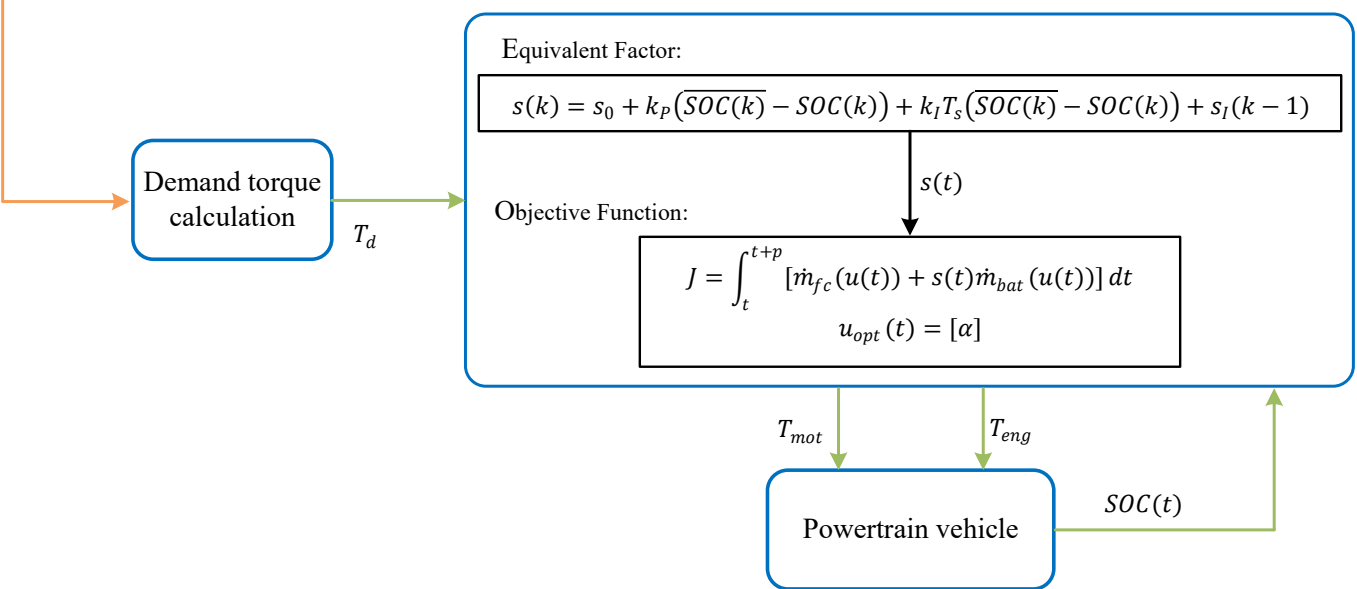

Figure 5. An illustration of the real-time power management in hybrid electric vehicles.

\subsection{Car-Following Layer}

In this section, the model-predictive-control-based multi-objective framework shown in Figure 5 is developed to achieve optimal overall performance by optimizing trade-offs between tracking safety and comfort during the car-following process. The car-following problem is formulated as a constrained nonlinear control system and is then solved by model predictive control. The following describes the performance indicators used for optimization. 


\subsubsection{Driving Safety}

As seen in the car-following scenario in Figure $1, v_{l}$ and $a_{l}$ represent the speed and acceleration of the leading vehicle, while $v_{f}$ and $a_{f}$ represent the speed and acceleration of the following vehicle. The expected distance between two vehicles $L_{d}$ can be expressed as

$$
L_{d}(k)=L_{0}+h v_{f}
$$

Here, $L_{0}$ represents the static inter-vehicle distance. $h$ represents the constant headway time.

The current actual distance $L(k)$ between the two vehicles can be expressed as

$$
L(k)=L_{0}+\sum_{k=1}^{T}\left(v_{l}(k)-v_{f}(k)\right) \Delta t
$$

Here, $T$ represents the current time.

Considering driving safety, the minimum following distance should be greater than the minimum braking distance [27]. The braking distance $L_{b r k}(k)$ can be calculated as

$$
L_{b r k}(k)=v_{f}(k) \tau_{b r k}+\frac{v_{f}(k)^{2}}{2 a_{b r k}}
$$

Here, $\tau_{b r k}$ represents the response time of the braking system, and $a_{b r k}$ represents the braking deceleration. The minimum distance between two vehicles $L_{\min }(k)$ can be expressed as

$$
L_{\min }(k)=L_{0}+L_{b r k}\left(v_{f}\right)=L_{0}+v_{f}(k) \tau_{b r k}+\frac{v_{f}(k)^{2}}{2 a_{b r k}}
$$

Here, $L_{0}$ is the static inter-vehicle distance and set as $2.4 \mathrm{~m}, \tau_{b r k}=0.5$ and $a_{b r k}=8$ according to [27], so

$$
L_{\min }(k)=2+0.5 \cdot v_{v e h}+0.0625 \cdot v_{v e h}^{2}
$$

For ACC systems, the maximum following distance can guarantee the traffic density to improve the road efficiency [3]. In this paper, the maximum following distance $L_{\max }(k)$ is related to the behavior of the driver and the braking distance. So, $L_{\max }(k)$ is expressed as

$$
L_{\max }(k)=L_{1}+L_{b r k}\left(v_{f}\right)=L_{1}+v_{f}(k) \tau_{b r k}+\frac{v_{f}(k)^{2}}{2 a_{b r k}}
$$

Here, $L_{1}$ is the dynamic inter-vehicle distance and is set as $10 \mathrm{~m} . \tau_{b r k}=1$ and $a_{b r k}=200 / 33$ according to [27], so

$$
L_{\max }(k)=10+v_{f}(k)+0.0825 v_{f}(k)^{2}
$$

In order to allow the following vehicle to reach the target speed and keep the following distance within a safe range, when the following distance is greater than the maximum following distance, the cost function will become very large. In order to ensure safety, the following distance cannot be less than the minimum following distance. Therefore, the cost function can be defined as

$$
J_{S}(k)= \begin{cases}\text { inf } & L(k)<L_{\min }(k) \\ b_{1}\left(L_{d}(k)-L(k)\right) & L(k) \in\left[L_{\min }(k), L_{d}(k)\right] \\ b_{2}\left(L(k)-L_{d}(k)\right)^{2} & L(t) \in\left[L_{d}(k), L_{\max }(k)\right] \\ b_{2}\left(L_{\max }(k)-L_{d}(k)\right)^{2}+b_{3}\left(L(k)-L_{\max }(k)\right)^{2} & L(k)>L_{\max }(k)\end{cases}
$$

Here, $J_{s}(k)$ is the cost of driving safety. $b_{1}, b_{2}$ and $b_{3}$ are cost coefficients, and it is necessary to ensure that $b_{3}$ is much larger than $b_{1}$ and $b_{2}$. For instance, $b_{1}, b_{2}$ and $b_{3}$ can be set to 1,2 and 50, respectively, based on [8]. 
The cost function is optimized with the SQP method, which utilizes the gradient of the objective function. The following function is utilized to approximate "inf":

$$
y=\frac{1}{L(k)-L_{\min }(k)+1}+\left(L_{d}(k)-L_{\min }(k)\right)
$$

For piecewise points, the gradient of the segment close to $L_{d}(k)$ is utilized.

\subsubsection{Vehicle Comfort}

Vehicle impact degree is an important parameter in the process of car-following, and its loss function is shown as follows:

$$
\begin{gathered}
j e r k=d a_{f} / d t \\
J_{\text {com }}= \begin{cases}h \cdot j e r k^{2}(k), & |j e r k|>1 \mathrm{~m} / \mathrm{s}^{3} \\
0, & |j e r k| \leq 1 \mathrm{~m} / \mathrm{s}^{3}\end{cases}
\end{gathered}
$$

Here, $h$ is a constant and is set as 0.1. The vibration of the vehicle body is inevitable in the process of traveling, so when the impact degree is set to be less than $1 \mathrm{~m} / \mathrm{s}^{3}$, the loss function is 0 . In addition, when the vehicle is not in a braking state, the maximum jerk should be less than $3 \mathrm{~m} / \mathrm{s}^{3}$.

\subsubsection{Overall Cost Function}

By combining driving safety with comfort, the instantaneous overall cost function $J$ is defined as

$$
J(k)=J_{s}(k)+J_{\text {com }}(k)
$$

This definition reflects the car-following dynamic performance within the prediction range of MPC in the form of a total cost function. In this paper, the ratio of driving safety factor and comfort factor is set to 1 according to [28].

The obtained optimized control input can be described as:

$$
u^{*}=\left[a_{f}^{*}\right]=\arg \min J
$$

\subsubsection{Optimization over the Moving Horizon}

The SQP algorithm is adopted to solve the objective function, and the optimized acceleration can be calculated. The velocity sequence obtained by the solution is as follows:

$$
\mathbf{v}_{\mathbf{f}}(\mathbf{j})=\left[v_{f}(j)+a_{f}(r) \Delta t, v_{f}(j)+2 a_{f}(r) \Delta t, \cdots, v_{f}(j)+a_{f}(r) l \Delta t\right]
$$

Here, the vector $\mathbf{v}_{\mathbf{f}}(\mathbf{j})$ is the speed sequence of the following vehicle over the $j$-th preview horizon, and $a_{f}(r)$ is the value of the specified allowable acceleration value in the grid.

Based on the optimal solution for each moving horizon, the first element of the optimal acceleration is taken as real input to update the vehicle dynamics, in accordance with the receding-horizon characteristic of MPC. The obtained speed sequence will be used for battery SOC prediction and used in the energy management strategy.

\subsection{Energy Allocation Layer}

In this paper, we present an energy management strategy for the hybrid electric vehicle based on MPC with ECMS. By applying the equivalent fuel consumption minimization algorithm to the MPC framework and using the ECMS algorithm to solve the MPC, an efficient instantaneous optimal control strategy is developed to optimize the allocation of torque and gear switching for a parallel HEV. The energy management strategy framework of a parallel HEV based on ECMS-MPC is shown in Figure 5. 
The ECMS energy control strategy is a transient optimization approach. The main idea is that the energy consumed by a parallel HEV during driving ultimately comes from the fuel consumption, and the power consumed by the power battery is supplemented later by a certain amount of fuel consumed by the engine during driving.

The MPC-based automotive energy management strategy optimizes the energy management of a parallel HEV by minimizing the predicted objective function in the time domain. The main optimization purpose of the energy management strategy in this paper is to minimize the fuel consumption of parallel hybrid electric vehicles. Therefore, the objective function can be expressed as the following formula:

$$
J_{\text {opt }}(k)=\sum_{t=k}^{k+N_{p}-1}\left(m_{f c}(u(k))+s(k) m_{\text {bat }}(u(k))\right) \cdot T_{s}, \quad k=1,2, \ldots, N_{p}
$$

Here, $m_{f c}(u(k))$ represents the engine fuel consumption, $m_{f_{c}}(u(k))$ represents the battery power. $u(k)$ is the control input and $N_{p}$ is the prediction time domain. $s(k)$ is the equivalent factor which reflects the variation of battery SOC. Frequent high DoD events are detrimental to battery health. To avoid DoD events, we try to maintain the battery SOC at a moderate level. If the SOC fluctuates greatly, the equivalent factor will increase and the weight of battery cost will increase in the joint optimization objective Equation (26), which will reversely punish the current control input $u(k)$ to return to a moderate SOC.

The system constraints are as follows:

$$
\left\{\begin{array}{l}
T_{r e q}(k)=T_{e}(k)+T_{m}(k) \\
T_{m_{-}} \min \leq T_{m}(k) \leq T_{m_{-}} \max \\
0 \leq T_{e}(k) \leq T_{e_{-} \max } \\
0 \leq n_{m}(k) \leq n_{m_{-} \max } \\
n_{e_{-} \min } \leq n_{e}(k) \leq n_{e_{-} \max } \\
S O C_{\min } \leq S O C(k) \leq S O C_{\max } \\
\alpha(k) \leq 1
\end{array}\right.
$$

Here, $T_{e}(k)$ and $T_{m}(k)$ represent the optimized engine torque and motor torque, respectively. $T_{m_{-} \min }$ and $T_{m_{-} \max }$ are the minimum torque and maximum torque. $n_{m}(k)$ corresponds to the current speed of the motor. $T_{e_{-} \max }$ is the maximum torque, and $n_{e}(k)$ corresponds to the current speed of the engine. $n_{m_{-} \text {max }}$ is the maximum speed of the motor. $n_{e_{-} \text {min }}$ and $n_{e_{-} \text {max }}$ are the minimum and maximum speed of the engine, respectively. $S O C_{\min }$ and $S O C_{\max }$ are the minimum and maximum values of battery SOC, respectively. $\alpha(k)$ is the ratio of the motor torque to the input demand torque.

To some extent, the future demand torque can be estimated from the generated speed sequence. In this paper, model predictive control is used to obtain the speed information in the predicted time domain $\left[k, k+N_{p}\right]$, so as to estimate the demand torque of the vehicle. Under the constraint conditions shown in (27), the optimal solution of the control variable is obtained by solving:

$$
\begin{gathered}
{\left[u_{o p t}(k)\right]=\underset{r \in\left[k, k+N_{p}\right]}{\arg \min }(J)} \\
u_{o p t}(k)=[\alpha(k)]
\end{gathered}
$$

The optimal solution of the control variable is the ratio of the motor torque to the input demand torque.

In the ECMS-based MPC energy management strategy, the selection of equivalent factors has a significant impact on the optimization results of the energy management strategy. In this paper, an adaptive regulation method using an equivalent factor based on the proportional integral (PI) form is adopted to change the automobile working condition. In contrast to the PI-equivalent factor proposed by [29], in this paper, to smooth 
the battery SOC further, a low-pass filtering mechanism is introduced to average the SOC. The proposed equivalent factor is as follows:

$$
\begin{gathered}
s(k)=s_{0}+K_{P}\left(S O C_{t a r}-\overline{\operatorname{SOC}(k)}\right)+K_{I} T_{S}\left(S O C_{t a r}-\overline{\operatorname{SOC}(k)}\right)+s_{I}(k-1) \\
\overline{\operatorname{SOC}(k)}=\frac{1}{p} \sum_{i=1}^{p} \operatorname{SOC}(k+i)
\end{gathered}
$$

Here, $s(k)$ represents the equivalent factor at time $t, \overline{S O C(k)}$ represents the mean of battery $S O C$ in the predictive time domain, $s_{0}$ represents the initial value of $s(k), K_{p}$ and $K_{i}$ are weight coefficients, $T_{s}$ is the sampling time and $s_{I}(k-1)$ represents the integral output at the previous moment $k-1$.

$S O C(k+i)$ is the battery's $S O C$ values at each time point in the generated speed sequence. The demand torque and motor power sequences can be obtained from the generated speed sequence by Equations (3) and (9). The battery SOC value in the time domain is calculated according to the torque ratio at the current moment $k$, as shown in the following:

$$
\frac{d(S O C)}{d t}=-\frac{1}{\eta_{\text {coul }}^{\text {sign }(I(t))} Q_{\text {nom }}}\left[\frac{V_{o c}(S O C)}{2 R_{0}(S O C)}-\sqrt{\left(\frac{V_{o c}(S O C)}{2 R_{0}(S O C)}\right)^{2}-\frac{P_{b a t t}}{R_{0}(S O C)}}\right]
$$

Here, $\eta_{c o u l}$ is the battery coulombic efficiency and $Q_{b a t}$ is the battery capacity. $V_{o c}$ and $R_{0}$ represent the battery open-circuit voltage and internal resistance, respectively.

Based on the ECMS-MPC control strategy, the future battery power consumption of an automobile in the finite time domain can be equivalent to fuel consumption, which reduces the difficulty of solving the algorithm and improves the calculation efficiency. In the case of unknown vehicle driving conditions, the above model predictive control is used to predict the vehicle speed in the predicted time domain, and the vehicle demand power is obtained according to the information of vehicle speed change, so as to optimize the control.

\section{Simulation Validation}

In this section, we evaluate the performance of the proposed energy management strategy with simulation results, implemented in MATLAB 2019b. The following vehicle consists of an engine, an electric motor, an automatic mechanical transmission and a battery pack. Specific parameters are listed in Tables 1 and 2.

Table 1. HEV configuration.

\begin{tabular}{cc}
\hline Components & Specifications \\
\hline Engine & FC-SI41 (Simulink) \\
& Maximum torque $T_{e_{-} \text {max }}: 170 \mathrm{Nm}$ \\
Electric machine & Maximum speed $n_{e_{-} \text {max }}: 5000 \mathrm{rpm}$ \\
& MC-PM30 (Simulink) \\
& Maximum power: $30 \mathrm{~kW}$ \\
& Maximum torque $T_{m_{-} \text {max }}: 200 \mathrm{Nm}$ \\
AMT & Minimum torque $T_{m_{-} \text {min }}: 200 \mathrm{Nm}$ \\
Battery pack & Maximum speed $n_{m_{-} \text {max }}: 6000 \mathrm{rpm}$ \\
& Gear ratio: $[1,4.212,2.637,1.8,1.386,0.772]$ \\
& ESS-NIMH6 (Simulink) \\
& Capacity, $Q_{b a t}: 5.3$ Ah $=19,080 \mathrm{C}$ \\
Coulombic efficiency, $\eta_{\text {coul }}: 0.95$
\end{tabular}


Table 2. Parameter settings in the simulation.

\begin{tabular}{ccc}
\hline Symbol & Characteristic (Unit) & Value \\
\hline $\mathrm{m}$ & Vehicle mass $(\mathrm{kg})$ & 1623 \\
$g$ & Gravitational constant & 9.81 \\
$C_{d}$ & Aerodynamic drag coefficient $(-)$ & 0.25 \\
$C_{r}$ & Rolling resistance coefficient $(-)$ & 0.9 \\
$\mathrm{~A}$ & Frontal area $\left(\mathrm{m}^{2}\right)$ & 2.46 \\
$\rho$ & Air density $\left(\mathrm{kg} / \mathrm{m}^{3}\right)$ & 1.29 \\
$r_{w}$ & Wheel radius $(\mathrm{m})$ & 0.34 \\
$i_{0}$ & Final drive ratio & 4.55 \\
$h$ & Constant headway time $(\mathrm{s})$ & 2.5 \\
$K_{p}$ & Weight coefficient & 3 \\
$K_{i}$ & Weight coefficients & 0.1 \\
$s_{0}$ & Initial value of equivalent factor & 4 \\
$T_{s}$ & Sampling time (s) & 1 \\
\hline
\end{tabular}

To validate the optimal performance of the ECMS-MPC energy management strategy, the simulation is implemented with standard cycles and the speed sequence generated in MATLAB.The proposed ECMS-MPC control strategy, the control strategy based on MPC and the control strategy based on MPC-DP are simulated and optimized respectively. Through the simulation analysis of the optimization results, the computational efficiency and the superiority of proposed strategy can be validated.

\subsection{Car-Following Performance under Different Driving Cycles}

In the simulation, the speed profile of leading car follows the NYCC driving cycle and UDDS driving cycle, as shown in Figure 6a,b. The optimized speed of the distance between leading vehicle and the following are shown in Figure 7.

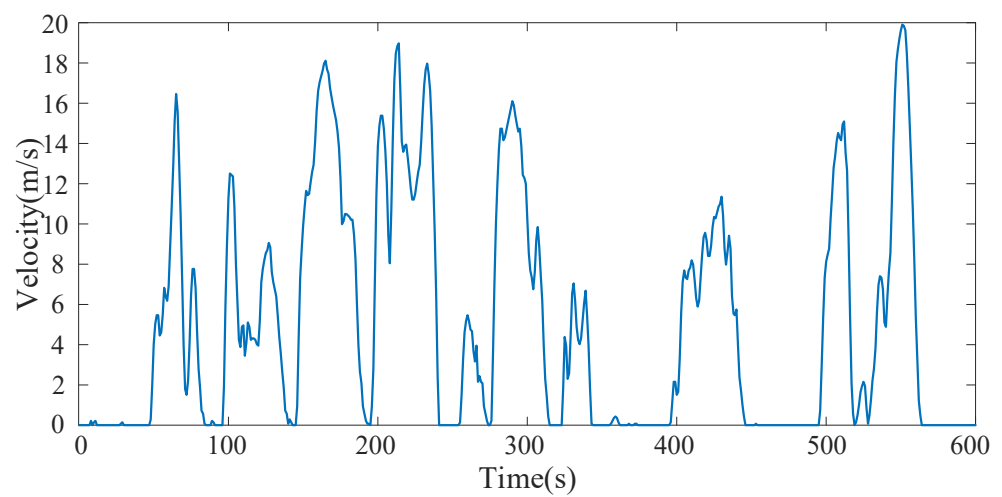

(a) The velocity profile of the NYCC driving cycle.

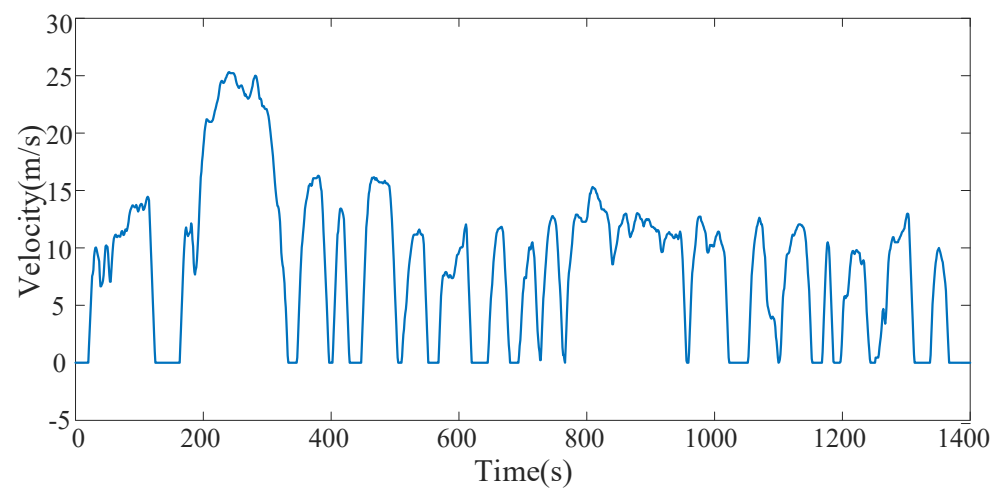

(b) The velocity profile of the UDDS driving cycle.

Figure 6. The NYCC driving cycle and the UDDS driving cycle. 
In Figure $7 \mathrm{a}$, the speed difference of the leading vehicle and the following vehicle under the NYCC driving cycle are presented. The optimized acceleration and deceleration of the following vehicle are presented in Figure $7 \mathrm{~b}$. The acceleration and deceleration are taken from the first value in the predicted time domain. The distance between the leading vehicle and the following vehicle are presented in Figure 7c. In the car-following results under the UDDS driving cycle in Figure $7 \mathrm{~d}-\mathrm{f}$, we can see clearly that the following vehicle follows the leading vehicle while ensuring a satisfactory inter-vehicle distance.
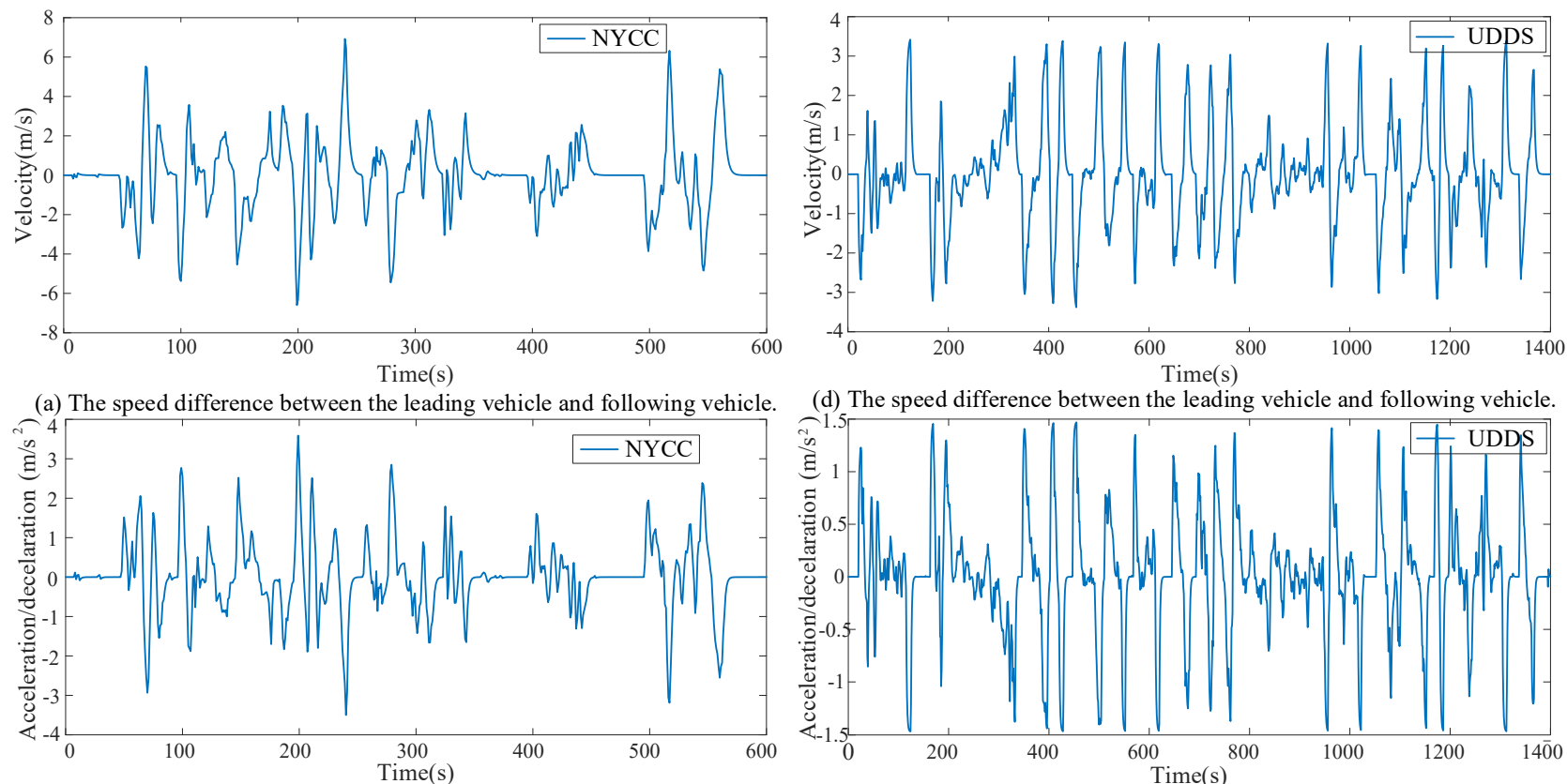

(b) The acceleration of the following vehicle.

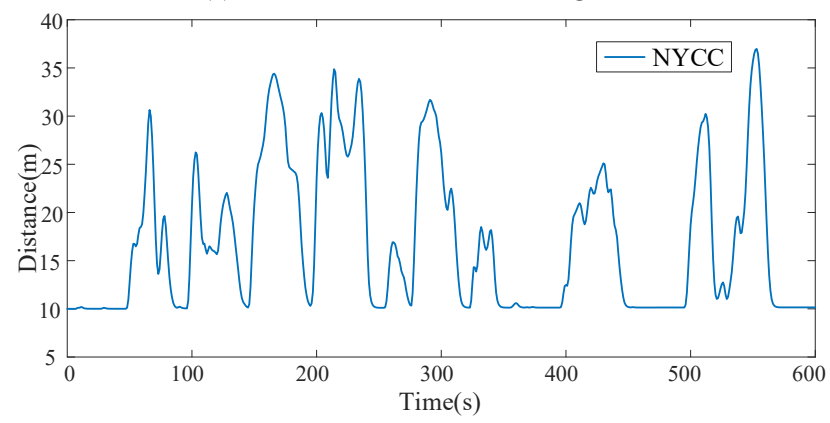

(c) The distance between the leading vehicle and following vehicle.

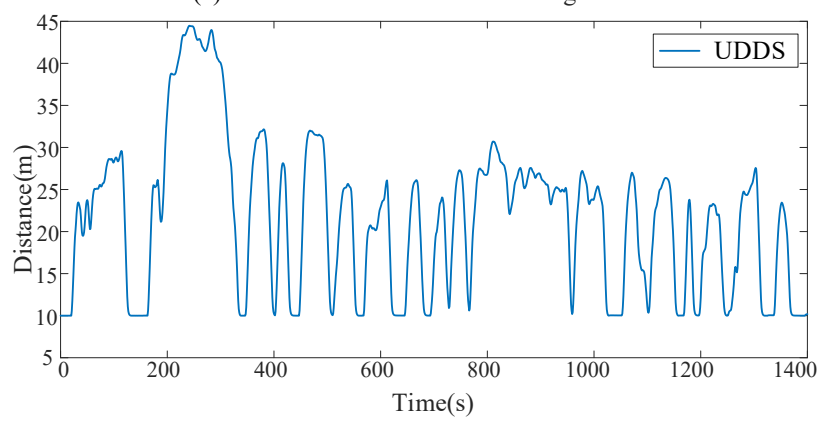

(f) The distance between the leading vehicle and following vehicle.

Figure 7. (a-c) The car-following results under the NYCC driving cycle. (d-f) The car-following results under the UDDS driving cycle.

\subsection{Energy Allocation Performance under Different Standard Driving Cycles}

The ECMS-MPC control strategy proposed in this paper is designed to achieve the energy management of a parallel HEV under the prediction of multi-objective optimization of vehicle speed prediction.

Therefore, in order to further evaluate the performance of the ECMS-MPC control method, the algorithm is used to optimize the energy management of a parallel HEV. NYCC and UDDS were selected to provide the tested driving cycles. The predictive time domain was chosen as $3 \mathrm{~s}$ and $5 \mathrm{~s}$, respectively according to the simulation result. In contrast to traditional MPC, due to the coupled effect of the car-following layer and the energy management layer, increasing the length of the time domain does not always increase the control performance. A longer preview horizon reduces the prediction accuracy of the future speed, which may result in increased power demands at the wheels. Second, for a 
longer preview horizon, more frequent emergency braking occurs, reducing the energy efficiency.

The equivalent factor is selected as 4 by trial and error. Its initial value is obtained by applying PMP and the shooting method. Then, depending on the difference $\operatorname{SOC}\left(t_{f}\right)-$ $S O C_{\text {target }}$, the initial value of the equivalent factor $s_{0}$ is increased or decreased in the next iteration, and the driving cycle is simulated again with a new initial value $s_{0}$. At the generic $n$th iteration, the value of $s_{0}$ is set to

$$
s_{0}(n)=\frac{1}{2}\left(s_{\text {inf }}(n-1)+s_{\text {sup }}(n-1)\right)
$$

Here, $s_{\text {inf }}$ and $s_{\text {sup }}$ are two variables introduced to implement a bisection method.

Taking the NYCC driving cycle as an example, the optimization results under DP and ECMS-MPC control strategies are shown in Figure 8. It can be seen from Figure 8a that the gear ratio of the two control strategies is basically the same, which illustrates the optimization of our proposed allocation strategy. From Figure $8 b, c$, it can be seen that the engine torque changes more frequently in the ECMS-MPC control strategy than in the DP control strategy, while the motor torque changes less frequently in the ECMS-MPC control strategy. There is a slight difference between the results of the ECMS-MPC and DP torque optimization. Because the DP is a global optimization method, the optimization results represent the global optimal; the ECMS-MPC is a local optimization method, and the optimization results represent local optimality. Therefore, there are some differences between the results of the two methods.

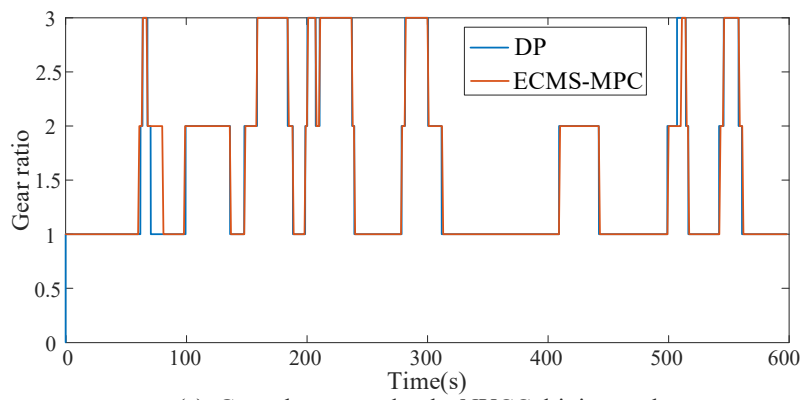

(a) Gear changes under the NYCC driving cycle.

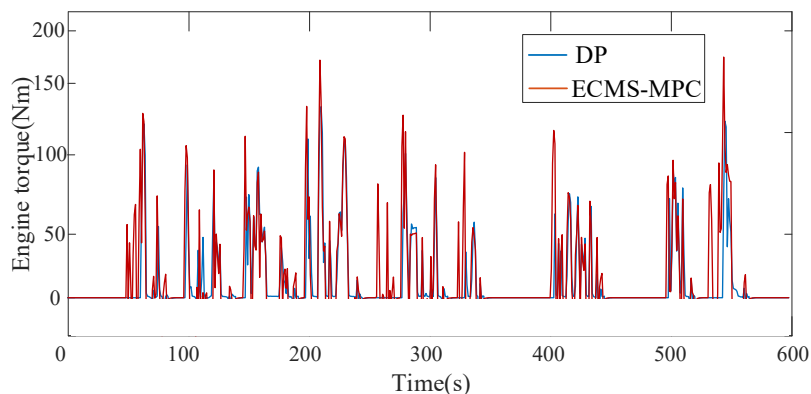

(b) The engine torque under the NYCC driving cycle.

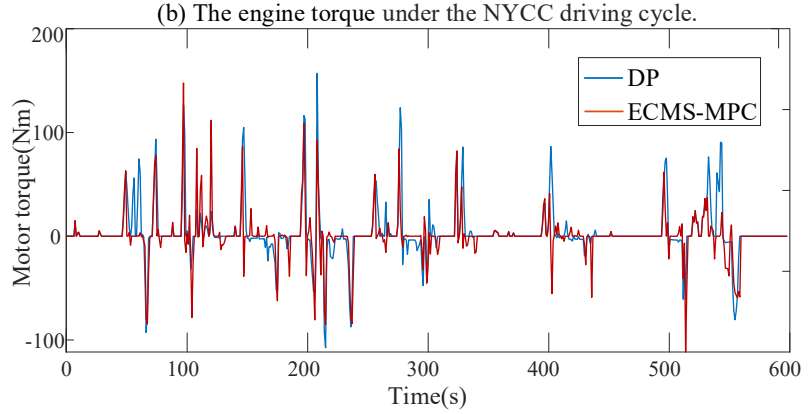

(c) The motor torque under the NYCC driving cycle.

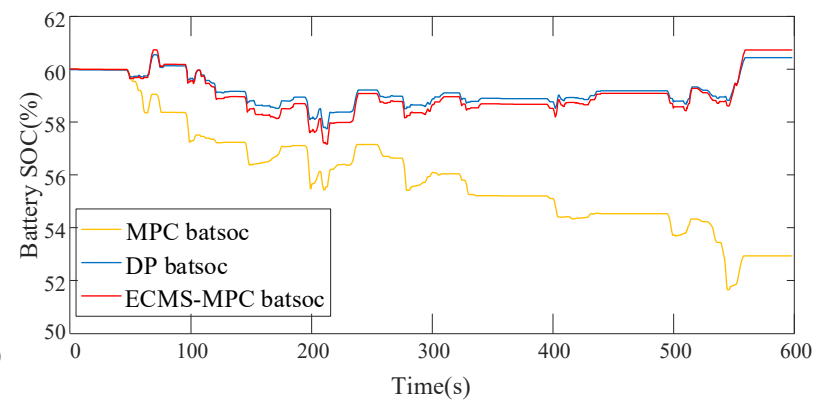

(d) The battery SOC comparison under different methods.

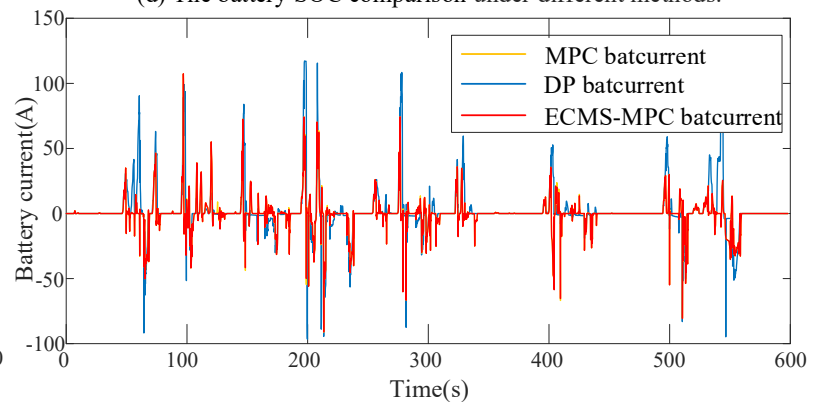

(e) The battery current comparison under different methods.

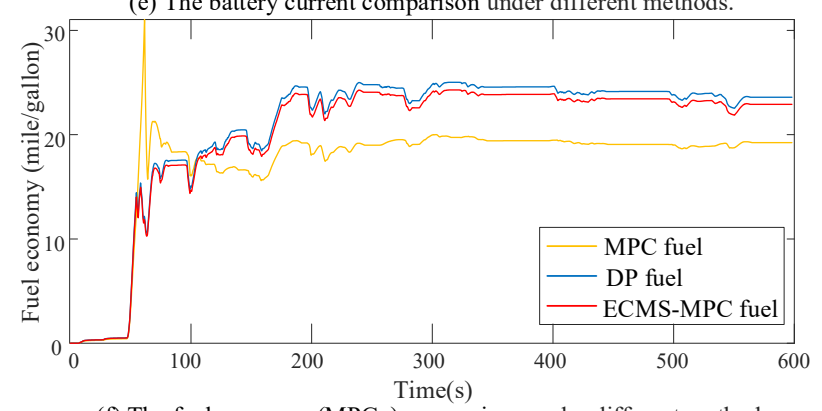

(f) The fuel economy (MPGe) comparison under different methods.

Figure 8. (a-c) The applied NYCC driving cycle and simulation in ECMS-MPC (5 s) and DP. (d-f) Comparison of the battery SOC and current when using different energy management strategies in the NYCC driving cycle. 
From Figure $8 d$, for all methods, the initial value of battery SOC is set as $60 \%$, which is also the expected final value. In the simulation, the tolerance is set as $\pm 5 \%$. In terms of SOC optimization, the global optimization method based on DP-MPC and ECMS-MPC could successfully maintain the stability of the battery SOC (Figure 8d).

In order to intuitively and effectively evaluate the optimization performance of ECMSMPC, a comparative simulation analysis is carried out with ECMS-MPC and DP respectively under two standard driving cycles. The fuel economy, computational efficiency and SOC sustainability of the ECMS-MPC control strategy are presented in Figure 8. In all simulations, the initial value of battery SOC is set as $60 \%$. In the case that the final value of the SOC cannot completely converge to the reference value, the adaptive equivalent factor transformation strategy is adapted. In terms of SOC optimization, the global optimization method based on DP-MPC and ECMS-MPC could successfully maintain the balance of battery SOC, as can be seen in Figure $8 \mathrm{~d}$. We can also see changes of the battery current in Figure 8e, and the ECMS-MPC control strategy still shows better results than the MPC control strategy. As can be seen from Figure 8f, in terms of fuel economy, compared with MPC, the fuel economy of ECMS-MPC in the NYCC driving cycle increases by about $2 \%$, while it is slightly lower than the result of DP because the optimization result of DP-MPC is globally optimal. These results are similar to those obtained with the UDDS driving cycle in Figure 9.

In order to better illustrate the optimization performance of ECMS-MPC, the MPC, DP and ECMS-MPC control strategies are used to optimize and compare under the predicted time domains of $3 \mathrm{~s}$ and $5 \mathrm{~s}$. The optimization results are shown in Tables 3 and 4 . In the tables, the calculation time of DP is the solution time of the whole cycle, and the calculation time of ECMS-MPC is the solution time in the predictive domain time. For the NYCC predicted conditions, it can be observed that the calculation time of ECMS-MPC in the time domain is $0.136 \mathrm{~s}$, and the calculation efficiency is much higher than that of DP. For the UDDS predicted conditions in different prediction time domains, ECMS-MPC also achieves the same calculation efficiency. In addition, with the increase of the prediction time domain, the calculation time of DP-MPC increases significantly, while the calculation time of the ECMS-MPC control strategy remains basically unchanged, indicating that this strategy allows optimization in a longer prediction time domain. Therefore, compared with DP, the ECMS-MPC energy control strategy has a higher computational efficiency and requires less computational effort in different prediction time domains, which is conducive to the realization of real-time automobile energy management optimization.

Table 3. Results of different controllers in the NYCC.

\begin{tabular}{cccccc}
\hline Method & FC (CNY) & EC (CNY) & TEC (CNY) & Final SOC & Time (s) \\
\hline MPC & 34.12 & 27.34 & 61.47 & 0.53 & 0.006 \\
ECMS-MPC (5 s) & 18.79 & 29.03 & 47.82 & 0.606 & 0.136 \\
ECMS-MPC (3 s) & 14.18 & 28.64 & 42.83 & 0.605 & 0.122 \\
DP & 10.10 & 29.13 & 39.24 & 0.603 & 17.79 \\
\hline
\end{tabular}

Table 4. Results of different controllers in the UDDS.

\begin{tabular}{cccccc}
\hline Method & FC (CNY) & EC (CNY) & TEC (CNY) & Final SOC & Time (s) \\
\hline MPC & 32.43 & 25.62 & 57.47 & 0.538 & 0.007 \\
ECMS-MPC (5 s) & 17.46 & 28.34 & 45.54 & 0.574 & 0.135 \\
ECMS-MPC (3 s) & 13.12 & 28.96 & 42.13 & 0.579 & 0.119 \\
DP & 9.28 & 29.34 & 38.72 & 0.596 & 28.36 \\
\hline
\end{tabular}




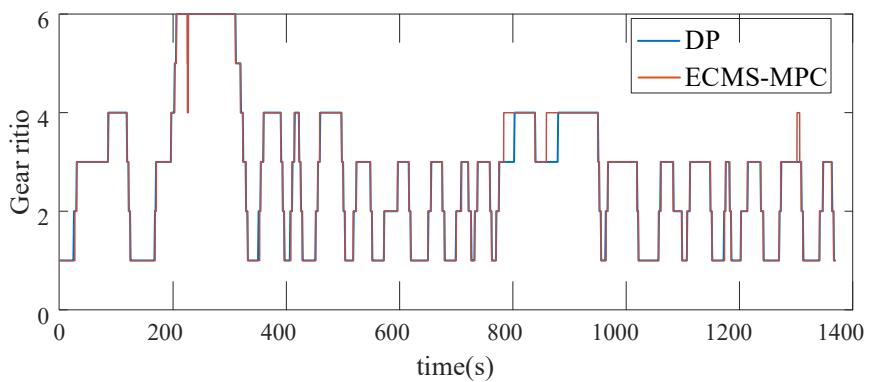

(a) Gear changes under the UDDS driving cycle.

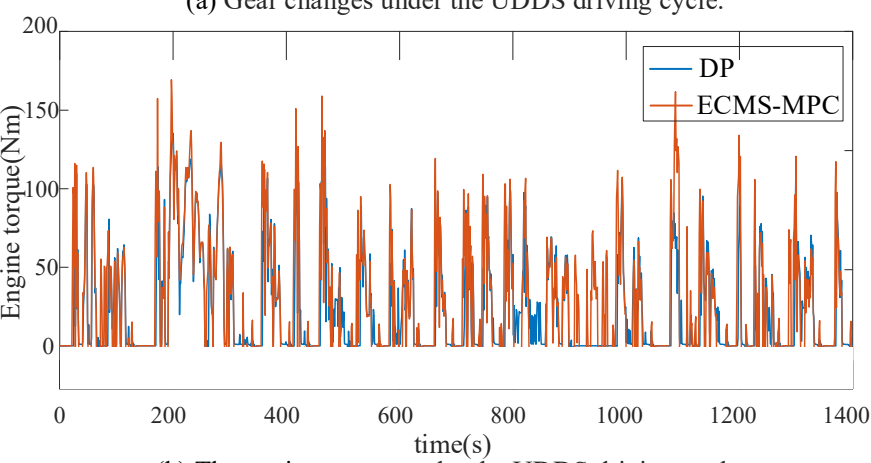

(b) The engine torque under the UDDS driving cycle.

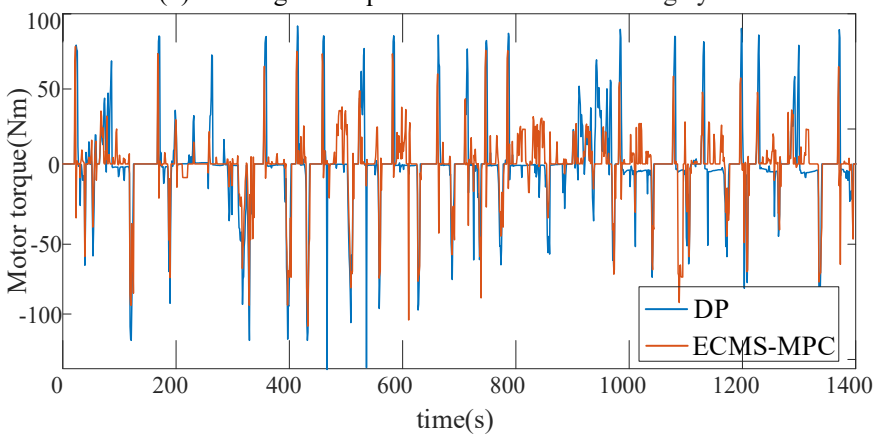

(c) The motor torque under the UDDS driving cycle.

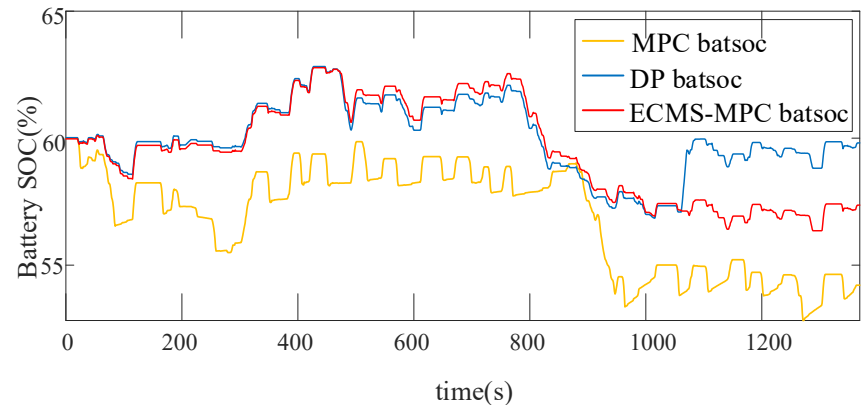

(d) The battery SOC comparison under different methods

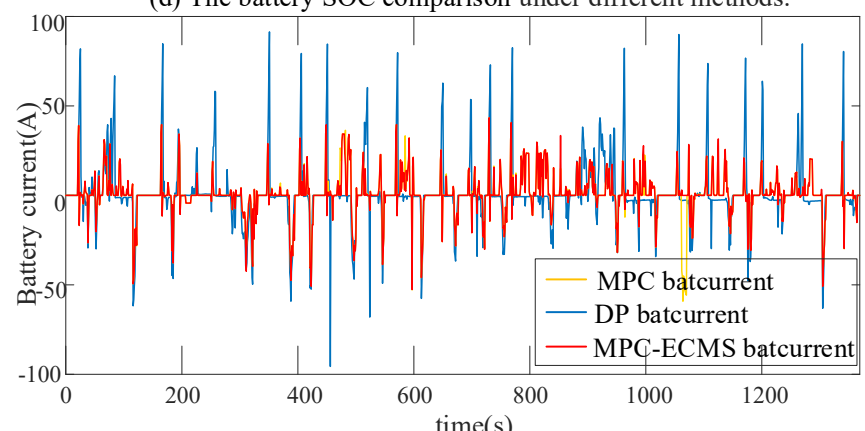

(e) The battery current comparison under different methods.

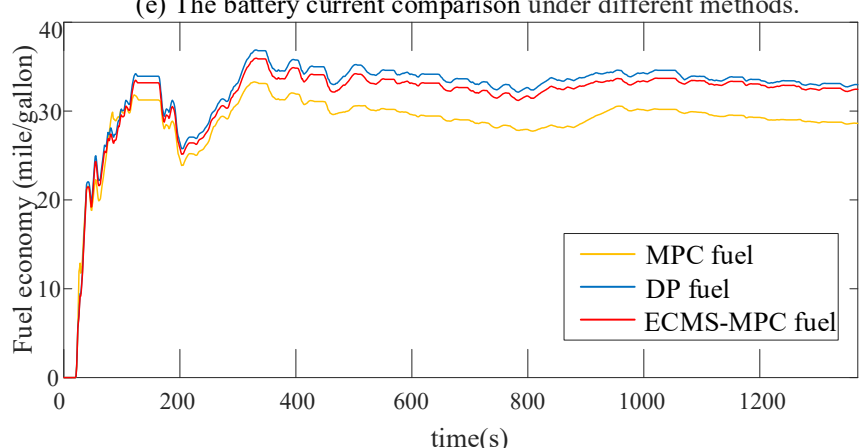

(f) The fuel economy (MPGe) comparison under different methods.

Figure 9. (a-c) The applied UDDS driving cycle and simulation in ECMS-MPC ( $5 \mathrm{~s})$ and DP. (d-f) Comparison of the battery SOC and current when using different energy management strategies in the UDDS driving cycle.

In Tables 3 and 4 , fuel cost (FC), electricity cost (EC), and total energy cost (TEC) are also compared for these methods. The fuel consumption of the ECMS-MPC algorithm is lower than that of the traditional MPC optimization algorithm. Under two driving cycles with different prediction time domains, the fuel consumption decreases by between $2.1 \%$ and $3.2 \%$. The fuel consumption of the ECMS-MPC algorithm is slightly higher than that of DP optimization algorithm because DP is a global optimization method, and can achieve the best fuel economy. However, the DP strategy requires the entire driving cycle to be known in advance. Therefore, although the fuel economy of ECMS-MPC is slightly worse, it does not need to know the operating conditions in advance, which is conducive to realtime optimization. For most of the predicted conditions, the fuel economy of ECMS-MPC is close to that of DP, and can maintain the balance of SOC for different predicted time domain conditions. In the UDDS driving cycle, compared with DP, ECMS-MPC could achieve a higher calculation efficiency, and the SOC has only a small deviation from the reference value, so the performance of ECMS-MPC is acceptable.

Based on the above contents, it can be concluded that the proposed ECMS-MPC energy management strategy can achieve a good performance in the test of different driving cycles. Additionally, its fuel consumption is close to that of the DP algorithm (which had the best fuel economy), confirming the performance of the control strategy in terms of torque distribution and simultaneously ensuring the balance of battery SOC. 


\section{Conclusions}

In this paper, an ECMS-MPC based energy management strategy for a parallel HEV is designed to address high fuel consumption and unbalanced battery SOC in the current energy management strategies, and an equivalent fuel consumption minimization algorithm is applied to the MPC framework. In order to improve the adaptability of the control strategy, the generated speed sequence based on car-following is established to obtain the vehicle speed information in the time domain. Then, combined with the predicted speed information, the optimal torque distribution control is realized using the ECMS energy management strategy. In order to verify the optimal performance of this control strategy, the effectiveness of this control strategy is verified by simulation and comparison with two other control strategies (i.e., DP and MPC) under different driving cycle sets, namely, NYCC and UDDS. In the simulation, our proposed method could reduce the fuel consumption by between $2.1 \%$ and $3.2 \%$, approaching the results of the DP method, which utilizes offline global optimization.

Author Contributions: Conceptualization, Z.H., W.L. and Y.W.; methodology, J.H. and X.S.; validation, M.W.; data curation, H.L. and X.S.; writing-original draft preparation, C.H. and X.S.; writing-review and editing, X.S. and W.L. All authors have read and agreed to the published version of the manuscript.

Funding: This research is supported by the National Natural Science Foundation of China (Grant Nos. 61672539, 61672537 and 61977062) and CERNET Innovation Project (Grant No. NGII20190603)

Institutional Review Board Statement: Not applicable.

Informed Consent Statement: Not applicable.

Data Availability Statement: Not applicable.

Conflicts of Interest: The authors declare no conflict of interest.

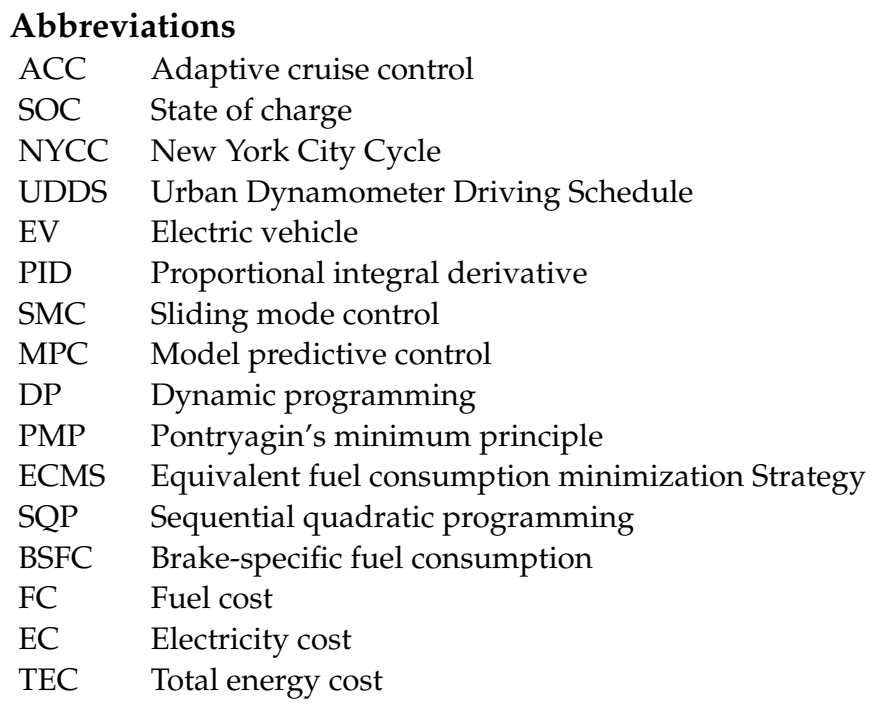

\section{References}

1. Shabbir, W.; Evangelou, S.A. Real-time control strategy to maximize hybrid electric vehicle powertrain efficiency. Appl. Energy 2014, 135, 512-522. [CrossRef]

2. Xu, F.; Shen, T. Look-Ahead Prediction-Based Real-Time Optimal Energy Management for Connected HEVs. IEEE Trans. Veh. Technol. 2020, 69, 2537-2551. [CrossRef]

3. Wu, D.; Zhu, B.; Tan, D.; Zhang, N.; Gu, J. Multi-objective optimization strategy of adaptive cruise control considering regenerative energy. Proc. Inst. Mech. Eng. Part D J. Automob. Eng. 2019, 233, 3630-3645. [CrossRef]

4. Zhang, X.; Huang, C.; Liu, M.; Stefanopoulou, A.; Ersal, T. Predictive cruise control with private vehicle-to-vehicle communication for improving fuel consumption and emissions. IEEE Commun. Mag. 2019, 57, 91-97. [CrossRef] 
5. Joševski, M.; Abel, D. Tube-based MPC for the energy management of hybrid electric vehicles with non-parametric driving profile prediction. In Proceedings of the 2016 American Control Conference (ACC), Boston, MA, USA, 6-8 July 2016; IEEE: Piscataway, NJ, USA, 2016; pp. 623-630.

6. Hussain, M.M.; Memon, Z.A.; Chaudhary, M.A.; Siddique, M. An Innovative PID Controller in Conjunction with DC Electric Motor for Control of Hybrid Electric Vehicle. Int. J. Electr. Electron. Eng. 2020, 7, 20-34.

7. $\mathrm{Hu}, \mathrm{X}$.; Wang, H.; Tang, X. Cyber-physical control for energy-saving vehicle following with connectivity. IEEE Trans. Ind. Electron. 2017, 64, 8578-8587. [CrossRef]

8. Xie, S.; Hu, X.; Liu, T.; Qi, S.; Lang, K.; Li, H. Predictive vehicle-following power management for plug-in hybrid electric vehicles. Energy 2019, 166, 701-714. [CrossRef]

9. Hu, X.; Zhang, X.; Tang, X.; Lin, X. Model predictive control of hybrid electric vehicles for fuel economy, emission reductions, and inter-vehicle safety in car-following scenarios. Energy 2020, 196, 117101. [CrossRef]

10. Xie, S.; Hu, X.; Xin, Z.; Li, L. Time-efficient stochastic model predictive energy management for a plug-in hybrid electric bus with an adaptive reference state-of-charge advisory. IEEE Trans. Veh. Technol. 2018, 67, 5671-5682. [CrossRef]

11. Li, L.; You, S.; Yang, C.; Yan, B.; Song, J.; Chen, Z. Driving-behavior-aware stochastic model predictive control for plug-in hybrid electric buses. Appl. Energy 2016, 162, 868-879. [CrossRef]

12. Zhang, F.; Hu, X.; Langari, R.; Cao, D. Energy management strategies of connected HEVs and PHEVs: Recent progress and outlook. Prog. Energy Combust. Sci. 2019, 73, 235-256. [CrossRef]

13. He, H.; Zhang, J.; Li, G. Model Predictive Control for Energy Management of a Plug-in Hybrid Electric Bus. Energy Procedia 2016, 88, 901-907. [CrossRef]

14. Banvait, H.; Anwar, S.; Chen, Y. A rule-based energy management strategy for plug-in hybrid electric vehicle (PHEV). In Proceedings of the 2009 American Control Conference, St. Louis, MO, USA, 10-12 June 2009; IEEE: Piscataway, NJ, USA, 2009; pp. 3938-3943.

15. Wang, D.; Lin, X.; Zhang, Y. Fuzzy logic control for a parallel hybrid hydraulic excavator using genetic algorithm. Autom. Constr. 2011, 20, 581-587. [CrossRef]

16. Li, G.; Görges, D. Ecological adaptive cruise control and energy management strategy for hybrid electric vehicles based on heuristic dynamic programming. IEEE Trans. Intell. Transp. Syst. 2018, 20, 3526-3535. [CrossRef]

17. Zeng, X.; Wang, J. A two-level stochastic approach to optimize the energy management strategy for fixed-route hybrid electric vehicles. Mechatronics 2015, 38, 93-102. [CrossRef]

18. Martinez, C.M.; Hu, X.; Cao, D.; Velenis, E.; Gao, B.; Wellers, M. Energy Management in Plug-in Hybrid Electric Vehicles: Recent Progress and a Connected Vehicles Perspective. IEEE Trans. Veh. Technol. 2017, 66, 4534-4549. [CrossRef]

19. Ali, A.M.; Söffker, D. Towards optimal power management of hybrid electric vehicles in real-time: A review on methods, challenges, and state-of-the-art solutions. Energies 2018, 11, 476.

20. Barsali, S.; Miulli, C.; Possenti, A. A control strategy to minimize fuel consumption of series hybrid electric vehicles. IEEE Trans. Energy Convers. 2004, 19, 187-195. [CrossRef]

21. Zhang, F.; Xi, J.Q.; Langari, R. An adaptive equivalent consumption minimization strategy for parallel hybrid electric vehicle based on fuzzy pi. In Proceedings of the 2016 IEEE Intelligent Vehicles Symposium (IV), Gothenburg, Sweden, 19-22 June 2016; IEEE: Piscataway, NJ, USA, 2016; pp. 460-465.

22. Xie, S.; Hu, X.; Qi, S.; Lang, K. An artificial neural network-enhanced energy management strategy for plug-in hybrid electric vehicles. Energy 2018, 163, 837-848. [CrossRef]

23. Xie, S.; Hu, X.; Qi, S.; Tang, X.; Lang, K.; Xin, Z.; Brighton, J. Model predictive energy management for plug-in hybrid electric vehicles considering optimal battery depth of discharge. Energy 2019, 173, 667-678. [CrossRef]

24. Zhang, F.; Xi, J.; Langari, R. Real-time energy management strategy based on velocity forecasts using V2V and V2I communications. IEEE Trans. Intell. Transp. Syst. 2016, 18, 416-430. [CrossRef]

25. Hussain, M.M.; Chaudary, M.A.; Razaq, A. Design and implementation of hybrid vehicle using control of DC electric motor. In Proceedings of the 2019 54th International Universities Power Engineering Conference (UPEC), Bucharest, Romania, 3-6 September 2019; IEEE: Piscataway, NJ, USA, 2019; pp. 1-6.

26. Hu, X.; Li, S.E.; Yang, Y. Advanced machine learning approach for lithium-ion battery state estimation in electric vehicles. IEEE Trans. Transp. Electrif. 2015, 2, 140-149. [CrossRef]

27. Li, L.; Wang, X.; Song, J. Fuel consumption optimization for smart hybrid electric vehicle during a car-following process. Mech. Syst. Signal Process. 2017, 87, 17-29. [CrossRef]

28. Luo, Y.; Chen, T.; Zhang, S.; Li, K. Intelligent hybrid electric vehicle ACC with coordinated control of tracking ability, fuel economy, and ride comfort. IEEE Trans. Intell. Transp. Syst. 2015, 16, 2303-2308. [CrossRef]

29. Onori, S.; Serrao, L.; Rizzoni, G. Equivalent Consumption Minimization Strategy. In Hybrid Electric Vehicles. SpringerBriefs in Electrical and Computer Engineering; Springer: London, UK, 2016. 